\title{
EXPERIENCE WITH THE COMISSIONING OF THE SUPERCONDUCTING STELLARATOR WENDELSTEIN 7-X
}

\author{
Hans-Stephan Bosch ${ }^{\mathrm{a}}$, V. Bykov ${ }^{\mathrm{a}}$, R. Brakel ${ }^{\mathrm{a}}$, P. van Eeten ${ }^{\mathrm{a}}$, J.-H. Feist ${ }^{\mathrm{a}}$, M. Gasparotto ${ }^{\mathrm{a}}$, H. Grote ${ }^{\mathrm{a}}$, T. Klinger ${ }^{\mathrm{a}}$,

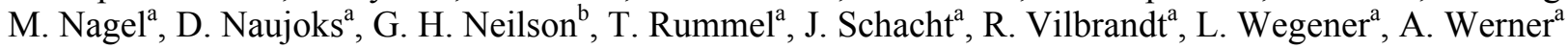 \\ ${ }^{a}$ Max-Planck-Institute for Plasma Physics, D-17491 Greifswald, Germany \\ ${ }^{b}$ Princeton Plasma Physics Laboratory, Princeton, NJ 08543, USA
}

\begin{abstract}
The super-conducting stellarator Wendelstein 7-X is presently under construction at the Max-Planck-Institute for Plasma Physics in Greifswald, Germany. Assembly of the device is almost completed and the periphery systems and the diagnostic and heating systems are well advanced. Commissioning of the device has been prepared over the last two years and has started in April 2014. This is the first time since decades that a superconducting fusion device is commissioned in Europe.
\end{abstract}

Keywords: Stellarator, Wendelstein 7-X, Commissioning

\section{Introduction}

After an extended time of design and fabrication of the Wendelstein 7-X (W7-X) stellarator[1, 2], the assembly of this devices comes to a close. W7-X is the first 'fully optimized' stellarator, which combines a quasi-isodynamic magnetic field configuration sustained by superconducting coils with a steady-state exhaust concept, steady-state heating at high power and a size sufficient to reach reactor relevant $n T \tau-$-values[3].

The W7-X configuration has a five-fold symmetry and therefore the device comprises five nearly identical magnet modules. The magnetic field configuration is described by a rotational transform $\mathrm{v} / 2 \pi$ of about 1 , i.e. in the range $0.72-1.25$, with low shear (i.e. a small variation of $1 / 2 \pi$ across the magnetic surfaces). The major radius of the plasma is $5.5 \mathrm{~m}$, the effective (i.e. averaged) minor radius is $0.55 \mathrm{~m}$, and the magnetic axis is helical. The machine height is $4.5 \mathrm{~m}$, the diameter is $16 \mathrm{~m}$. The total mass of the basic device (without diagnostics and heating devices) is 725 tons, the cold mass (superconducting coils, central support ring, inter-coil support elements, bus-bars and pipes) amounts to 425 tons.

After the start of machine assembly in 2005, about 900.000 man hours have been performed inside the assembly work. Assembly of the basic device is coming to an end rather soon. In June 2013 the last weld on the torus (inner and outer vessel) has been closed and in April 2014 the last of the 14 current leads [4] has been installed at the torus. With this action, the cryostat consisting of the outer vessel, the inner vessel and the ports in between - has been closed and the commissioning could start.

Parallel to these works, the installation of the invessel components has been continued [5] and will be finished in November 2014: The plasma vessel walls are covered with normal-graphite tiles on $\mathrm{CuCrZr}$-structures in the inner side and stainless steel cushions in the outer side of the plasma vessel (both components are foreseen for water-cooling, but are not cooled in the first operation phase 1.1). For this first operation phase, no divertor will be installed, but 5 graphite limiters are installed in the five modules in the bean-shape crosssection of the plasma 6].

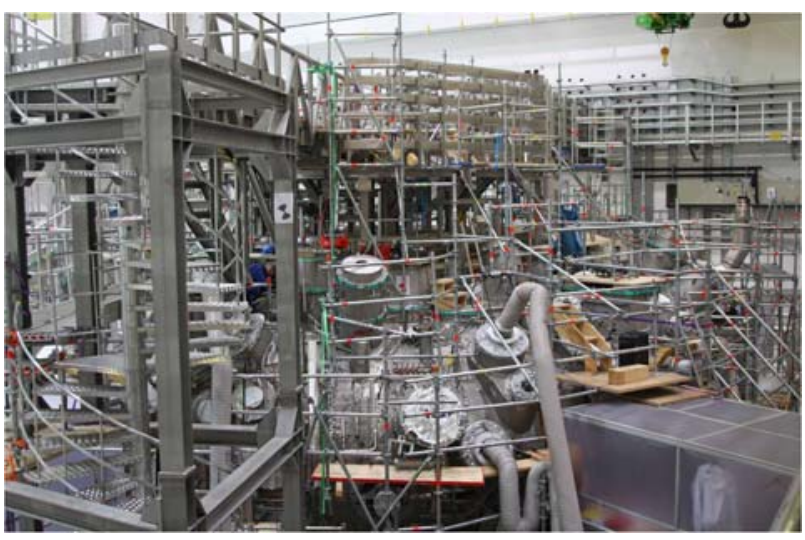

Fig. 2. Wendelstein 7-X in May 2014. On the left side, the so-called "Thomson bridge", which will support different diagnostics elements and allows access to the inner side of the torus is visible. On top of the device cable trays are mounted.

The most important work package in this phase of the assembly is concerned with the peripheral systems, first of all, the experiment platform [7] installed in 2013, but also a complex support tower in the center of the stellarator which supports all the cryo-lines coming from the basement to the stellarator, water cooling circuits, cable trays and other elements. This tower also allows access to the centre of the stellarator. Water cooling circuits are also installed, including the lines from the pumps in the basement to the torus, the ring lines below the torus, but also the very detailed distribution from the ring lines to the cryostat vessel (for cooling of the ports and diagnostics), or the feed-throughs through the 
cryostat (for cooling the outside of the plasma vessel) or to the plug-ins into the plasma vessel interior (for cooling the in-vessel components, the glow discharge electrodes or diagnostics). Although the in-vessel components will not be water-cooled in the initial operation phases 1.1 and 1.2, the water-lines in the torus hall (but also within the plasma vessel) have to be installed now for space-reasons.

Due to the tight space environment inside the torus hall, the platform, support frames, water lines and cable trays had to be designed simultaneously [8], and the assembly of these peripheral systems demands extreme logistical efforts. Due to the interaction of these components also during assembly, the assembly schedule is very sensitive and is prone to delays. Only at the very end of these steps, the cable trays can be fixed to the platforms and the support structures, and then the cables can be laid out and connected to the components, power supplies and computers.

However, since the cryostat is closed, commissioning has been started, and this paper describes the preparation and processes (section 2), the relevant $\mathrm{CoDaC}$ activities (section 3), the results of the commission activities up to now (section 4) and an outlook (section 5). In section 6 we discuss lessons learned in the start of commissioning.

\section{Preparation of W7-X commissioning}

In 2012 a Task Force started to detail the commissioning processes $[9,10]$. In general, our commissioning process for the $\mathrm{W} 7-\mathrm{X}$ is divided into two steps of increasing levels of system integration.

1) The local commissioning (LC) of a single technical component: The instrumentation and all other peripheral components are included as required, and the component is run by its local control system.

2) The integrated commissioning (IC) handles the step-wise integration of all separate components into the overall system $\mathrm{W} 7-\mathrm{X}$ including the central data acquisition and storage system and the central device control.

The sequence of commissioning W7-X can be divided into six phases, which can be treated separately, as they build up a sequence [9]. Only some tasks in this sequence can be performed in parallel, but the main process has to follows a serial sequence:

1. Vacuum tests of the cryostat

2. Cryogenic tests of the cryostat

3. Normal conducting coil systems tests

4. Vacuum tests of the plasma vessel

5. Superconducting magnet coil systems tests

6. Preparation for the first plasma

In the summer of 2013, after these processes for the commission of W7-X had been presented to an international workshop of experts, the commissioning group was set up, the formal processes were defined, and the technical detailing of the six phases mentioned above, was started (sequentially).

\subsection{Organization of the Commissioning}

The process instructions for the commissioning of W7-X have been set up according to the best engineering standards and are described in [11].

For each commissioning (local or integrated) the responsible officer for a system (RO) has to prepare a commissioning instruction, the so-called Commissioning Assurance Template (CAT), which contains all necessary work and test steps in the right sequence. The CAT is based on the technical, functional or project specification of the system and the instruction manual as well as the safety analysis.

An operation manual must be prepared for each system. It must contain the description of all relevant technical details, a description of the intended use of the system and the instructions for the putting into service. Instructions for the training of operators and for shortterm maintenance must be added if necessary.

The CAT must include the necessary input documents for each commissioning step and the corresponding checks to confirm the achieved states. Hold points for the verification of achieved operational states must be defined. On arriving at hold points, current states shall be compared with previously planned states. Furthermore, it must be checked if all conditions and requirements are fulfilled to allow the continuation of commissioning. Test protocols and check lists serve to verify exact examinations.

Based on the operation manual, the CAT and the safety analysis, the Health \& Safety group prepares a special health and safety briefing. They monitor the briefing of all the personal working in the torus hall. It should be noted, that the commissioning is performed in parallel to the final phases of the assembly.

\subsection{Management of the Commissioning}

The W7-X Project Board appoints the responsible Chief Commissioning Manager (CCM), having overall responsibility for all local and integrated commissioning. The CCM coordinates all activities such as planning, performance, evaluation and he approves all relevant documents. He is supported by the division head "Assembly", who coordinates simultaneously ongoing activities in the torus hall and other assembly areas.

The CCM is the head of the Central Commissioning Group (CCG). Members of the group are the heads of CoDaC, group leader "Machine Safety", division head "Assembly", the Health \& Safety group (or staff members who were appointed by them). The subdivision heads (TBL), responsible for the system currently under commissioning, are also members of the CCG to provide the full competence. The positions of chief mechanical 
and electrical engineers are foreseen for later commissioning phases and shall be appointed later.

The members of the CCG organize and supervise the execution of commissioning and the entire documentation from the instruction manuals to the final reports. The CCM is directly responsible to the $\mathrm{W} 7-\mathrm{X}$ Project Board. Urgent problems can be cleared also in meetings of the Configuration Control Board in which the scientific requirements are represented by the Scientific Director of W7-X. Strategic decisions are made in the W7-X Project Board.

The CCG is supported by staff members of the technical groups involved in the commissioning processes (Cryogenics, Cryostat, Magnet System, Vacuum Systems, Diagnostics, and Heating departments,...). Necessary work contributions from other groups shall be organized and controlled by the $\mathrm{CCM}$ or the responsible members of the CCG.

The responsibility for the preparation and execution of both commissioning steps of a relevant system shall remain with the present $\mathrm{RO}$ or the $\mathrm{RO}$ who shall also be in charge of later operations.

\section{Role of CoDaC in W7-X commissioning}

Control, Data acquisition and Communication (CoDaC) [12] plays a special role in the commissioning process, as all components have to be controlled (locally) and simultaneously integrated into the central control system. This is an important aspect of the IC, which cannot be tested before. Therefore, it is a rather complex system which controls components, also with regard to safety aspects, but it also is commissioned in the same time. Also, the technical data from the commissioning process have to be archived from the beginning in order to have all detailed information for later analyses.

The architecture of the Wendelstein 7-X control and data acquisition system is based on a distributed control and data acquisition system [13], in which each component (like diagnostic systems, heating devices and auxiliary systems) is equipped with a local control system: Local Commissioning of any component is performed using this local control and local data acquisition. The Integrated Commissioning also involves the Central Control System.

In fact, Central Device Control consists of two different systems:

1. The Central Operation Management System, cOPM, which is responsible for operational management and sequence control (glow discharge, baking, ...) for different operational phases of the device. The main function of the COPM is to guide and control the global behavior of the W7-X machine. The hardware of the cOPM is based on proven industrial techniques of Co. Siemens / Germany [14]. The controller of the cOPM is a very powerful Programmable Logic Controller (PLC), type S7-416-H. The interfaces between the cOPM and all components of the experiment $\mathrm{W} 7-\mathrm{X}$ is made by a network connection of type Profibus-DP. The software of the cOPM is developed with software tool Process Control System PCS7 (Co. Siemens). For the human machine interface (HMI) a redundant server client system is used. The software for the visualization is developed with Co. Siemens software tool Window Control Center (WinCC).

2. The Central Safety System cSS, which handles all requirements for the personnel safety as primary requirement and also investment protection (device safety) controlled by the Central Interlock System. The cSS consists of two main parts, Safety Control System (SCS), and the Fast Interlock System (FIS). The SCS realizes personnel and machine safety tasks with reaction times above $50 \mathrm{~ms}$. Central Safety functions with requires reaction times below $50 \mathrm{~ms}$ down to $1 \mathrm{~ms}$ are implemented inside the FIS. The safety system of W7-X uses special certified safety hardware. The safety program of the cSS runs in a redundant PLC system with two CPUs S7-416-H (Co. Siemens). Via a safety bus, based on a Profisafe protocol, all decentralized safety interfaces of W7-X technical components and diagnostics are connected to the cSS. The HMI is also based on a server client system. In addition, a hardware control console serves as a backup system in case of a fault of the computer HMI system.

The definition of safety functions of the personnel and plant safety purposes are strictly related to the German and European regulations, e.g. the rule DIN EN 61511 (Functional safety) and EN 62061 (Safety of machines).

The CoDaC systems will be put into operation synchronized to the commissioning plan. Since each component is equipped with a local control system, this system is used for the LC and commissioned within this process. As far as available in this phase, the data acquisition system is being used to archive all the engineering data produced by that component. The programmable logic controller in a local system sends the data to a dedicated data acquisition station 24 hours each day. All the data will be archived in the central Experiment Data Archive. Collecting data, in particular for the machine instrumentation in the early phases, will facilitate error analysis and document the mechanical and thermal behavior of the complex device for comparison with later operation phases.

Correlated actions of different components will be introduced step by step into the control system in order to support the IC and to keep the tests of central systems at low complexity.

In a later stage of the IC, the discharge control system based on segment control with a real time system [15] will be taken into operation. This system supports fast correlated actions, e.g. where fast camera data acquisition has to be synchronized with mechanical 
actuators. This phase also prepares the first plasma operation.

\section{Recent results of $W 7-X$ commissioning}

With the closure of the cryostat in March 2014, i.e. after the installation of the last current leads, the commissioning started. While all electrical connections inside the cryostat have been tested after each assembly step, a global electrical (insulation) test of these connections has been performed before the cryostat was closed.

As discussed already in $[9,10]$, the commissioning process started with vacuum tests of the cryostat, which includes two different systems:

a) The interspace-vacuum system (IVS), pumping the interspaces of the multilayer port bellows [16], the double sealed flanges at the outer vessel and on the plasma vessel as well as the combined water- and current feeding system for the control coil, had to be commissioned and the leak tightness of these bellows interspaces, flanges and the vacuum piping in between had to be confirmed. Especially the multilayer port bellows have to be pumped to a pressure of at least 100 mbar, before the cryostat can be pumped down, because these bellows cannot stand a pressure difference of 1 bar.

The IVS consists of 5 pumping stands, each one serving one module of W7-X and consisting of two rotary vane pumps, ten branches equipped with individual valves and measuring gauges and about $115 \mathrm{~m}$ of vacuum piping up to and on the outer vessel. During the local commission of the IVS, leaks in this vacuum piping have been found, dust and flakes found in some of the pipes required the addition of filters in all subcircuits, and the local control system for the pumping stands required some modifications. But, all these repairs and modifications were quickly implemented, and the multilayer bellows were commissioned by June 2014 , thereby allowing the evacuation of the cryostat.

b) The cryo-vacuum system (CVS), pumping the cryostat (the space formed by the outer vessel, the inner vessel and the 254 ports in between) also consists of five pumping stations, each one containing a fore-vacuum system (a rotary vane pump and a roots blower, about 10 $\mathrm{m}$ away from the outer vessel) and a turbo-molecular pump $3.5 \mathrm{~m}$ below the outer vessel. Due to tight space conditions in the torus hall the design of the control cubicles and their cabling were somewhat delayed and therefore the setting up of the control system for the pumping stations was carried out in stages. By mid of July two of the CVS fore-vacuum system were ready, two weeks later all 5 systems were in this status, in early August the first turbo-molecular pump was ready and since end of August all five pumping stands are fully commissioned, i.e. all 15 pumps are working with their valves and instrumentation included in the local control and with cOPM.
The IC of the cryostat vacuum started mid of July with the first evacuation of the cryostat. This was performed in a condensed concentrated effort over a weekend to avoid interferences with the ongoing assembly work in the torus hall. This evacuation was not only the integrated commissioning of the cryo-vacuum pumping system, and the start of central data acquisition, but also the acceptance trial for the cryostat as a vacuumcontainer. Therefore, in parallel to the evacuation (see 4.1) also the mechanical and structural behavior of the vessels and ports were monitored and investigated (see 4.2). The data from the pressure gauges in the cryostat and all the mechanical measurements were collected in the central W7-X data base and plotted and interpreted with the W7-X browser or via a web-interface.

\subsection{Evacuation of cryostat}

Figure 1 shows the development of the cryostat vessel pressure during the first evacuation of the W7-X cryostat performed with two rotary vane pumps. The plateaus shown in this figure have been implemented to perform the mechanical measurements described in the next section. In these phases, the pumps have been closed off, and one can clearly see a pressure rise due to leaks.

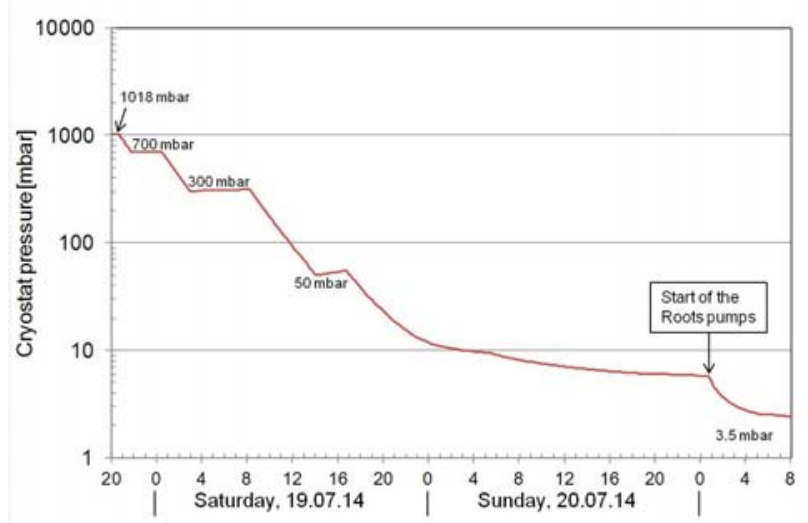

Fig. 2. Course of the pressure in the W7-X cryostat during the first evacuation. The plateaus at 700, 300 and 50 mbar were introduced to perform mechanical test/measurements on the cryostat.

The pump down started rather fast (1 Hour to the first plateau), but the evacuation speed decreased continuously. Below a pressure of about 20 mbar, water in the cryostat became dominant and the pressure decrease became rather slow. Only on Monday morning 21.07, the pressures in the cryostat got below 5 mbar and the roots blowers started.

\subsection{Structural investigations of the cryostat}

Evacuating the cryostat introduces mechanical loads and stresses on the shell structure, resulting in deformations of the vessels and ports and relative movement between the vessels. Of special interest are the port bellows, specifically those bellows from large oval and rectangular ports where significant deformation is expected due to the vacuum load. 
To investigate the deformations/deflections of the cryostat, a finite element (FE) model has been set up, the "global model of the cryostat system" (GMCS) [17, 18]. This model has been used to perform detailed calculations for different load cases (cryostat and PV evacuation, PV baking, normal operation with PV and OV evacuated, etc.). Recently an updated version of the GMCS (v3.4) was used to predict the cryostat behavior during its first evacuation and to verify this prediction with a set of different measurements performed during this first evacuation [19].

These calculations have shown that most pronounced deformation values (up to $3.5 \mathrm{~mm}$ ) are expected in the upper part of the machine. Moreover, pressure drops of 0.2 bar should add displacements of approx. $0.5 \mathrm{~mm}$, which are well measurable. It was expected that displacement and stress levels behave quasi linearly in relation to pressure during cryostat evacuation. Therefore it was considered useful to perform measurements at predefined pressure levels, see fig. 2. Laser trackers were used to measure the deformation of the cryostat and of ports at 42 reference points. Figure 3. Shows, as an example, the predicted and the measured results for four reference points on supply ports as a function of the pressure in the cryostat (the history goes from right (1 bar) to the left, i.e. vacuum conditions). Most of the 42 reference points showed good agreement with the prediction from the FE modelling. About 10 out of the 42 measured points, both on OV and on supply ports, showed stronger deviations, all being still within a maximum displacement of 3.3 $\mathrm{mm}$. In general the cryostat behaved as expected.

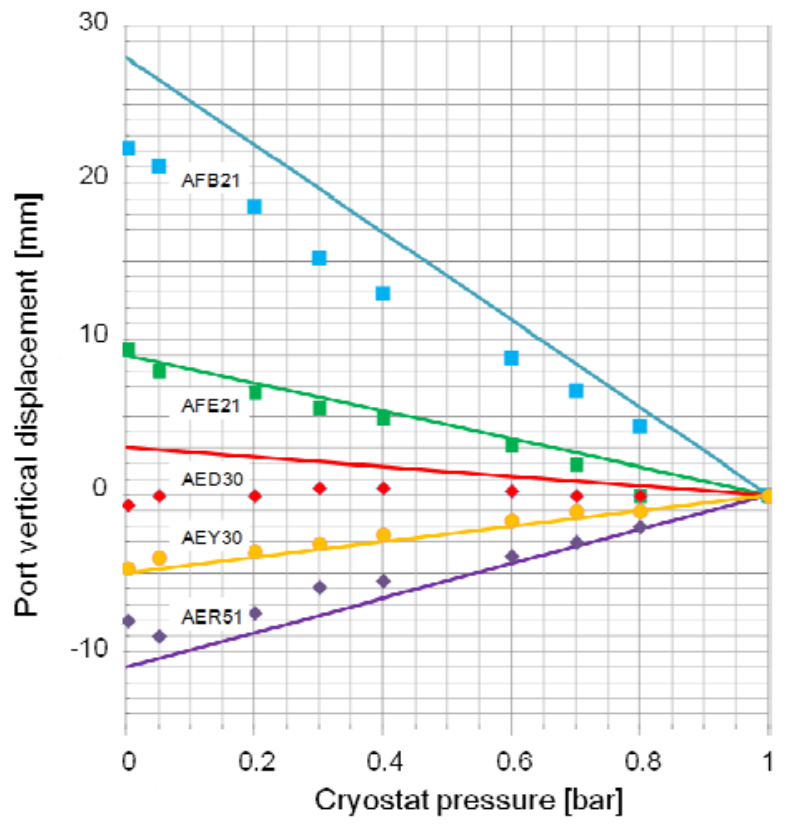

Fig. 3. Displacement of typical ports during the first evacuation (pressure decreasing during the campaign)[19]. The solid lines indicate the values calculated by the FE model, the corresponding symbols show the values measured with a laser tracker.
Another prediction of the GMCS dealt with the strain on the plasma vessel. The response of the cryostat and its supports to the vacuum loads is basically linear. This was confirmed by the results of the PV strain gauges, but also by the plasma vessel support load and port bellow deformation measurements. All of these measurements showed reasonable agreement with the predictions from the GMCS [19].

\subsection{Vacuum conditions in the cryostat}

As was discussed in the chapter 4.1, the first evacuation was performed with only two pumping units. A few weeks later, the other three units became operational, and soon after that, the turbomolecular pumps were integrated. Since beginning of September, all 5 turbomolecular pumps are operating continuously.

During the evacuation in July five leaks on port welds have been detected, which have been welded after a venting of the cryostat with dry air. After the following evacuating further leak search with He-spraying was continued and increasingly smaller leaks were detected and repaired. As of today the base pressure in the cryostat (measured with low conductance between the cryostat wall and the thermal insulation) is $1 \times 10^{-3} \mathrm{mbar}$ ( $1.5 \times 10^{-4}$ mbar in front of the turbomolecular pumps), and the total gas rate is $0.6 \mathrm{mbarl} / \mathrm{s}$, including roughly $0.5 \mathrm{mbarl} / \mathrm{s}$ from outgassing of the walls and the multilayer insulation (mainly water).

\section{Further steps in W7-X commissioning}

As mentioned before, the integrated commissioning of the cryostat is almost finished, the leak search is continuing further.

The next phase concerns the cryo-supply, i.e. the cryo plant, and the He-cooling circuits in the cryostat. The local commissioning of the cryo-plant has been performed in 2013, but the cooling circuits still have to be commissioned. This step involves the cleaning and purging of the cooling circuits and has been started end of September, see figure 4 for the commissioning schedule. The integrated commission will start in January 2015 with the cool-down of the magnet system (superconducting coils, central support ring, inter-coil supports and cryolegs) and the tests of three operation modes, defined by their operating temperature:

1. Long Stand-by $(\mathrm{T}=100 \mathrm{~K})$

2. Short Stand-by $(\mathrm{T}=10 \mathrm{~K})$

3. Normal operation $(\mathrm{T}=4 \mathrm{~K})$ for operating the coils up to $2.5 \mathrm{~T}$.

Also in October we will start the commissioning of the trim coils [20] and later of the control coils inside the plasma vessel. 


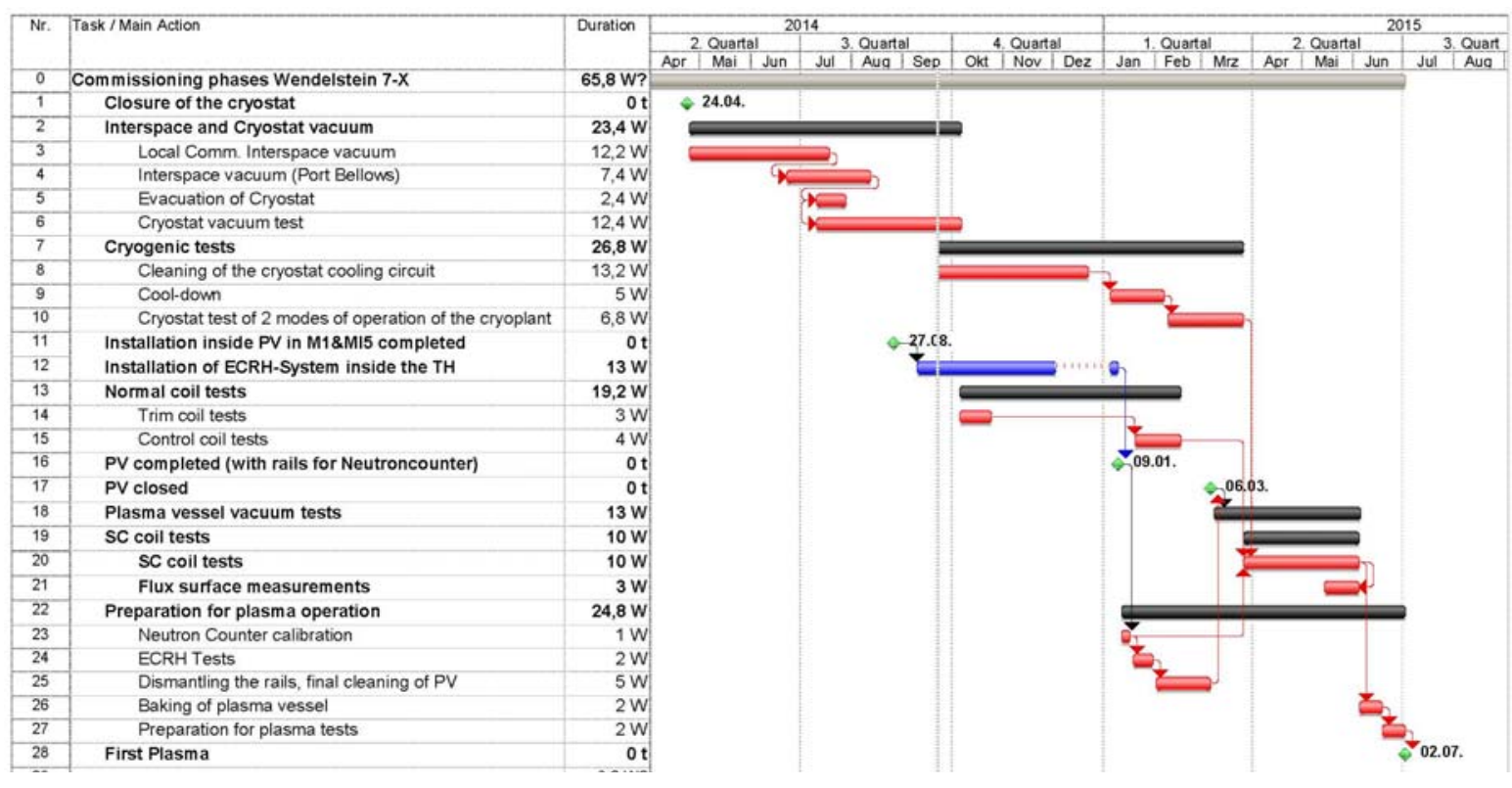

Fig. 5. Schedule for W7X-commissioning with the status of September 2014

In January 2015, the assembly work in the plasma vessel is finished and the neutron counters can be calibrated, with a neutron source moving on a rail in the center of the plasma vessel to simulate a toroidally extended neutron source. After a last adjustment of the ECRH mirrors, the rail can be dismantled and the plasma vessel will be cleaned and finally closed.

At this moment the plasma vessel can be commissioned (pumped out, confirmation of leak tightness).

After the end of the cryo-supply tests, the commissioning of the superconducting coils will start. First, each of the 7 coils circuits (5 non-planar and 2 planar coil types) will be loaded with currents up to the nominal values, later the whole magnet system will be operated simultaneously, thereby creating the confining magnetic flux surfaces. At this point in time we will measure the flux surfaces simultaneously [9].

Parallel to the tasks on the major device systems, the most important diagnostics systems, the control systems and the ECRH system are commissioned.

\section{Conclusion}

Commissioning of Wendelstein 7-X has started after the cryostat vessel was closed, i.e. it is running in parallel to the last assembly steps. This has imposed logistic difficulties and has implied additional efforts to secure personnel safety. However, no problems with the safety issues have been observed from this interplay.

Commissioning requires lots of preparation, also with papers on safety analysis and safety hazards, operation manuals, and commissioning templates. This means additional burden for the team members, but it

has proven important to prepare the commission carefully.

While preparing the commissioning of the major systems mentioned previously, it is very important to monitor also the status of the so-called periphery, e.g. cooling circuits (for water, liquid $\mathrm{He}$, gas exhaust), cables and control systems. These are important systems, and must be always in the focus.

For commissioning tasks that involve several systems (like evacuation of the cryostat) a trial run (in which the pressure in the cryostat has been lowered by 50 mbar) has proven to very valuable. Such a trial will also be implemented for the cool down.

\section{Acknowledgments}

This work has been carried out within the framework of the EUROfusion Consortium and has received funding from the European Union's Horizon 2020 research and innovation programme under grant agreement number 633053. The views and opinions expressed herein do not necessarily reflect those of the European Commission.

\section{References}

[1] J. Nührenberg et al., Overview on Wendelstein 7-X theory, Transactions on Fusion Technology 27 (1995) 71.

[2] T. Klinger et al., Towards Assembly Completion and Preparation of Experimental Campaigns of Wendelstein 7-X in the Perspective of a Path to a Stellarator Fusion Power Plant, Fusion Engineering and Design 88 (6-8) (2013) 461-465.

[3] H.-S. Bosch et al., Technical challenges in the construction of the steady-state stellarator Wendelstein 7X, Nuclear Fusion 53 (12) (2013) 126001. 
[4] T. Rummel et al., The current leads of the Wendelstein 7$\mathrm{X}$ superconducting magnet system, IEEE Transactions on Plasma Science 42 (3) (2014) xxx-xxx.

[5] I. Boscary, Design and technological solutions for the plasma facing components of Wendelstein 7-X, Fusion Engineering and Design 86 (6-8) (2011) 572-575.

[6] Bozhenkov, S. A. et al., Limiter for the early operation phase of W7-X, Proc. 41st EPS Conf. on Plasma Physics, June 2014, Berlin

[7] S. Renard et al., Design and Integration of the Ground Level Platform for W7-X, IEEE Transactions on Plasma Science 42 (3) (2014) TPO-130.

[8] C. Baylard et al., Concurrent Engineering and Mechanical Integration in the Experimental Hall of Wendelstein 7-X: Issue, Solutions, Lessons Learned, IEEE Transactions on Plasma Science 42 (3) (2014) Th06-03.

[9] H.-S. Bosch et al., Transition from Construction to Operation Phase of the Wendelstein 7-X Stellarator, IEEE Transactions on Plasma Science 42 (3) (2014) 432-438.

[10] M. Gasparotto et al., "Wendelstein 7-X-Status of the project and commissioning planning", Fusion Engineering and Design 89 (2014) 2121-2127.

[11] R. Vilbrandt et al. Continuity and Enhancement of Quality Management during commissioning of W7-X, this conference.

[12] A. Werner et al., Cutting Edge Concepts for Control and Data Acquisition for Wendelstein 7-X, Proceedings of the $25^{\text {th }}$ Symposium on Fusion Engineering (SOFE), San Francisco, June 2013.

[13] J. Schacht et al., Task and structure of the Wendelstein 7$\mathrm{X}$ control system, Fusion Engineering and Design 81 (2006) 1799-1806.

[14] J. Schacht et al., Stellarator WEGA as a test-bed for the WENDELSTEIN 7-X control system concepts, Fusion Engineering and Design 83 (2008) 228-235.

[15] H. Laqua et al., Control system of Wendelstein 7-X experiment, Fusion Engineering and Design 66-68 (2003) 669-673.

[16] J. Reich et al., Manufacture of the plasma vessel and the ports for Wendelstein 7-X, Fusion Engineering and Design, 75-79 (2005) 565-569.

[17] V. Bykov et al., "Specific Features of Wendelstein 7-X Structural Analyses", IEEE Transactions on Plasma Science, 42 (3) (2014) .

[18] A. Tereshchenko et al., "FE simulation of the Wendelstein 7-X cryostat system", Fusion Engineering and Design 84 (2009) 1833-1837

[19] P. van Eeten et al., „Features and Analyses of W7-X Cryostat System FE Model”, SOFT2014, P3.007

[20] T. Rummel at al., The Wendelstein 7-X trim coil system, IEEE Transactions on Applied Super-conductivity 24, (3) (2014) 4200907 (4pp.). 